\title{
A MODULAR OPTICS DESIGN FOR THE NUMI BEAMLINE
}

\author{
JOHN A. JOHNSTONE
}

Fermilab, Batavia $\amalg 60510$

$$
\text { May } 28^{\text {th }}, 2002
$$

Protons extracted from the Main Injector in the MI60 straight are transported $374 \mathrm{~m}$ to the NuMI target. This $120 \mathrm{GeV} / \mathrm{c}$ transfer line design is comprised of 4 major sections: an optical matching section between the Injector and transport line; a section of periodic FODO cells; a special insertion fashioned after the Tevatron Collins straights to bridge the $68 \mathrm{~m}$ carrier pipe drift, and; a tunable final focusing module to achieve the desired beam parameters on the target. Horizontal bending is provided by EPB dipoles, while vertical bends are produced (predominantly) by B2 dipoles. The optical characteristics are defined by 21 quadrupoles of the 3Q60/120 series.

Three Lambertsons and a C-magnet at Q608 in the MI extract the beam upwards at an angle of 27.3 $\mathrm{mr}$. By powering the 2 Lambertsons downstream of Q608 20\% above their nominal $120 \mathrm{GeV} / \mathrm{c}$ fields sufficient separation is created between the extracted and MI circulating beams to insert a rolled (900) 3Q120 magnet $^{1}$ into the slot between the C-magnet and MI quad Q609.

Six rolled EPB's, each bending by $11.5 \mathrm{mr}$, steer the beam onto its final horizontal trajectory while also leveling it off $0.90 \mathrm{~m}$ above the MI, but below the Recycler Ring. This is the only horizontal bend in the line. Another 70 meters downstream 6 vertically-bending B2's pitch the beam down through the carrier pipe at an angle of $156 \mathrm{mr}$. A final set of 4 B2's remove $98 \mathrm{mr}$ of this vertical angle; aligning the beam between the target $30.5 \mathrm{~m}$ further downstream and the Soudan detector.

\begin{tabular}{|c|c|c|c|c|c|}
\hline \multicolumn{5}{|c|}{ DIPOLES } & \multirow{2}{*}{ Roll } \\
\hline String & Type & \# & $\begin{array}{l}\text { Length } \\
\text { (m) }\end{array}$ & $\begin{array}{c}\mathrm{B} \\
(\mathrm{T}) \\
\end{array}$ & \\
\hline HV1 & EPB & $\begin{array}{l}1 \\
1 \\
3 \\
1\end{array}$ & 3.048 & 1.5085 & $\begin{array}{r}0.00^{\circ} \\
22.28^{\circ} \\
32.63^{\circ} \\
22.28^{\circ}\end{array}$ \\
\hline V2 & B2 & 6 & 6.0706 & 1.7148 & $90^{\circ}$ \\
\hline V3 & B2 & 4 & 6.0706 & 1.6103 & $90^{\circ}$ \\
\hline
\end{tabular}

Table 1. Relevant parameters of the 3 major bend centers.

[ Design fields : $\mathrm{EPB}=1.5 \mathrm{~T} @ 1.688 \mathrm{kA}: \mathrm{B} 2=1.8 \mathrm{~T} @ 4.750 \mathrm{kA}$ ]

\footnotetext{
1 The $3 \mathrm{Q} 60 / 120$ series quads are normally installed in the configuration with a $\mathrm{H}$ x W cross-section of $17^{\prime \prime} \times 13^{\prime \prime}$. Rolling the magnet through $90^{\circ}$ gains $2 "$ of vertical clearance for free.
} 


\section{$\underline{\text { Optics }}$}

Lattice functions for the transfer line are illustrated in Figure 1. Here, the final focus quadrupoles are tuned to produce a spot size of $\sigma_{\mathrm{x}}=\sigma_{\mathrm{y}}=1.0 \mathrm{~mm}$ at the target for a $40 \pi(95 \%$, normalized) emittance beam. Quadrupole parameters are listed in Table 2.

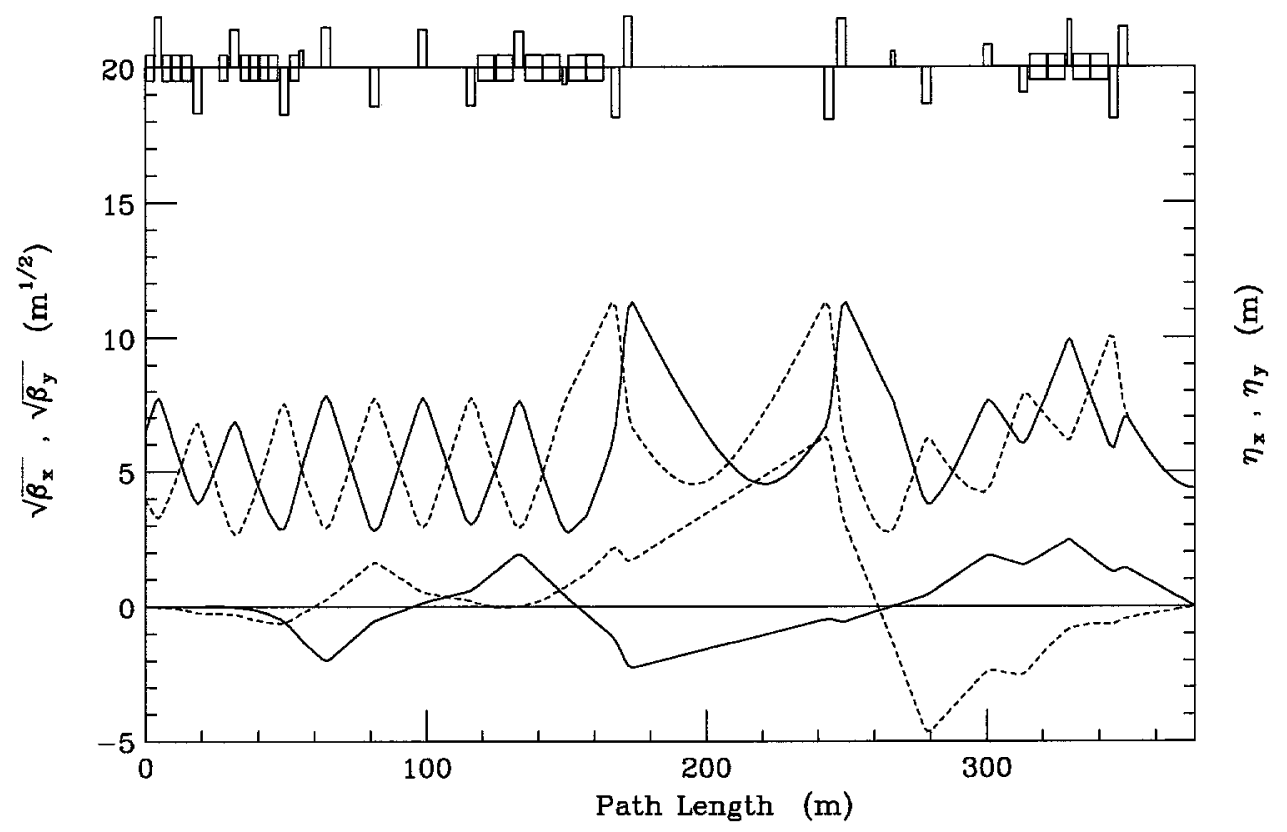

Figure 1. Horizontal (solid) \& vertical (dashed) lattice functions of the NuMI transfer line. The first 4 magnets shown are in the MI - the Lambertsons, C-magnet, and quad Q608.

\begin{tabular}{||c|c|c|}
\hline \multicolumn{3}{|c||}{ QUADRUPOLES } \\
\hline \multicolumn{3}{|c|}{$\begin{array}{c}\text { Length } \\
(\mathrm{m})\end{array}$} \\
\hline Quad \# & $\begin{array}{c}\text { Gradient } \\
(\mathrm{T} / \mathrm{m})\end{array}$ \\
\hline Q101 & 3.048 & 14.2316 \\
\hline Q102 & 3.048 & 12.9414 \\
\hline Q103 & 3.048 & 14.6570 \\
\hline Q104 & 1.524 & 5.4057 \\
\hline Q105/106/107/108/109 & 3.048 & 12.3445 \\
\hline Q111/115 & 1.524 & 5.2681 \\
\hline Q112/113 & 3.048 & 15.6431 \\
\hline Q116 & 3.048 & 16.3658 \\
\hline Q117 & 3.048 & 11.3899 \\
\hline Q118 & 3.048 & 7.2141 \\
\hline Q119 & 3.048 & 7.8995 \\
\hline Q121 & 1.524 & 15.7905 \\
\hline & 3.048 & 16.1907 \\
\hline & 3.048 & 12.6893 \\
\hline
\end{tabular}

Table 2. Quadrupole circuits and parameters.

[ Design fields=18.9 T/m @ $100 \mathrm{~A}$ ] 
The first 4 quadrupoles in the transfer line are powered individually and perform the optical match between the appropriate $\beta$ 's \& $\alpha$ 's of the MI to those of the transfer line. Through this section $\beta$ is kept small enough in both planes to avoid problems with the tight EPB apertures. The matching section is followed by $86 \mathrm{~m}$ of FODO cells characterized by quads Q105 $\rightarrow \mathrm{Q} 109$. The half-cell length and phase advance are such that this closely replicates the MI lattice. Quads Q110, Q111, \& Q112 at the upstream end of the carrier pipe, and Q113, Q114, \& Q115 at the downstream end, create long straight section optics. The peak $\beta$ 's in the doublets are equal at $\beta(\max ) \approx 125 \mathrm{~m}$.

The last 6 quadrupoles in the line form the final focus optics to obtain the desired beam size at the target and also to eliminate horizontal \& vertical dispersion at the target. With only 1 horizontal bend center in the line it is not possible to also achieve $\eta_{\mathrm{x}}=0$. And, with the additional vertical constraints imposed by the existing tunnel, neither is it simple to fix $\eta_{y}=0$ over any appreciable range of extracted MI beam emittances. With $\beta_{\mathrm{X}}(\max ) \& \beta_{\mathrm{y}}(\max ) \approx 100 \mathrm{~m}$, aperture restrictions are not an issue through this region. The final focus segment is optically flexible and can comfortably accommodate tuning to at least $\pm 30 \% \beta$ variations in each plane independently.

Magnet apertures \& beam envelopes appear in Figure 2. One contour reflects the MI beam parameters as extrapolated to the NuMI era ${ }^{2}$, when emittance will be $\varepsilon=40 \pi \mathrm{mm}-\mathrm{mr}$ (95\%, normalized) $\& \delta \mathrm{p}_{95} / \mathrm{p}=7 . \mathrm{E}-4$, for Gaussian distributions. The much more important result, though, is the envelope calculated for $\varepsilon=500 \pi(100 \%$, normalized $) \& \delta \mathrm{p}_{100} / \mathrm{p}=28$.E-4.

The $500 \pi$ maximum transverse emittance is determined by the restricted space seen by the circulating MI beam as it passes the Lambertson magnets. In the arcs, where dispersive contributions inflate the beam size, a $500 \pi$ emittance coupled with a momentum spread of $\delta \mathrm{p}_{100} / \mathrm{p}$ $\approx \pm 7$.E-3 completely fills the machine aperture and, therefore, dcînes the MI admittance. This admittance value for the momentum spread, however, is not a sensible value to use in NuMI tracking studies. The RF bucket area at $120 \mathrm{GeV} / \mathrm{c}$ is $\sim 10 \mathrm{eV}-\mathrm{sec}^{3}$, whereas a $\delta \mathrm{p}_{100} / \mathrm{p}=7 . \mathrm{E}-3$ translates into a longitudinal emittance of $\sim 50-60 \mathrm{eV}$-sec and so could not possibly be accelerated - it simply doesn't fit in the bucket. The maximum spread that can be contained by the RF is $\delta \mathrm{p}_{100} / \mathrm{p}$ of $\sim 2.8 \mathrm{E}-3$.

The real significance of the $500 \pi \& \delta \mathrm{p}_{100} / \mathrm{p}=2.8 \mathrm{E}-3$ envelope in Figure 2, therefore, is that it demonstrates that the NuMI line should be able, without losses on the magnets, to transport the worst quality beam that the Main Injector can conceivably spew forth.

\footnotetext{
2 S. Mishra, "Main Injector Performance During NuMI Operations", presented at the NuMI Beam Optics Director's Review, April, 2002.

${ }^{3}$ M.J. Syphers, "Some Notes on Longitudinal Emittance", internal Fermilab report, April, 2002.
} 


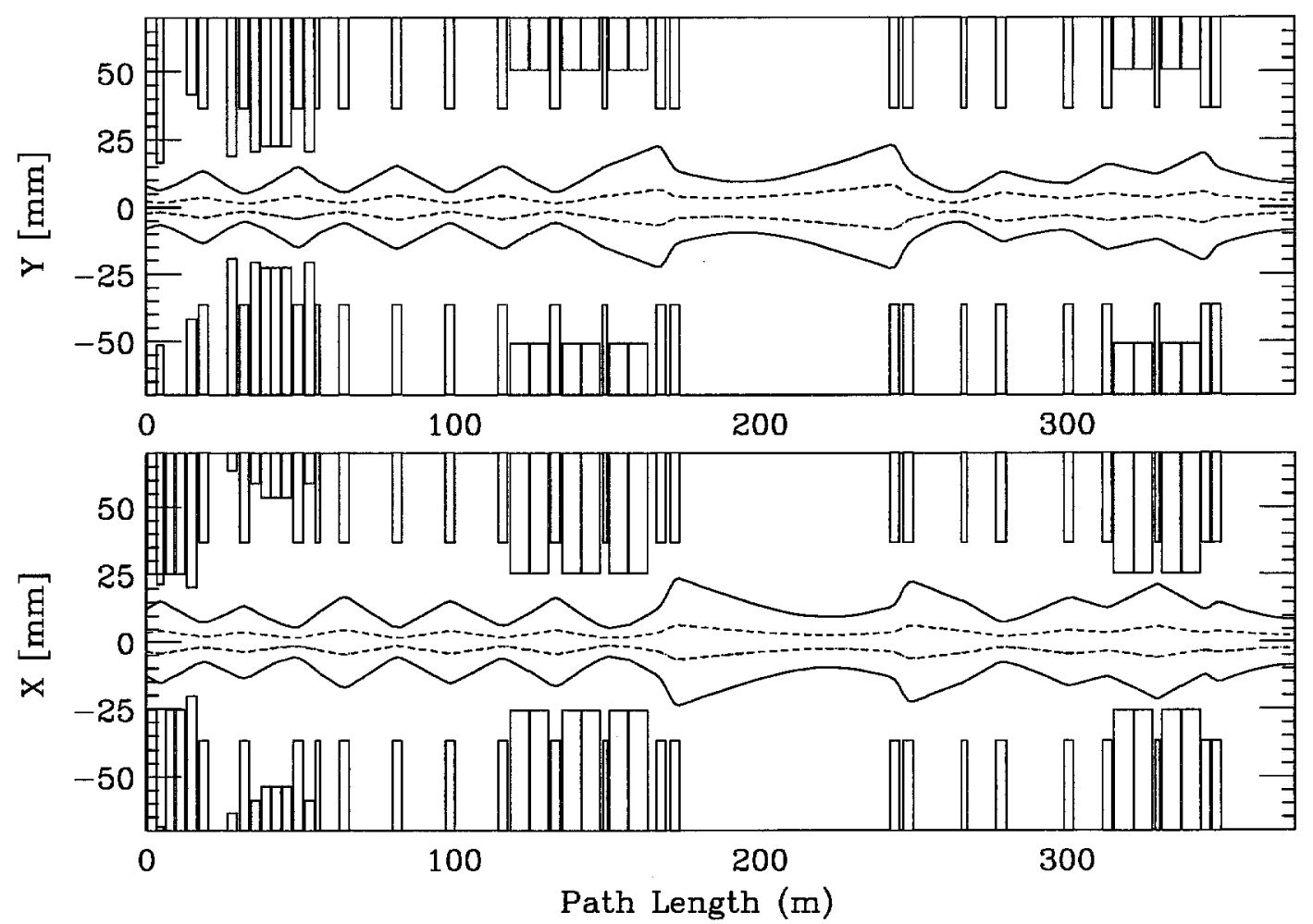

Figure 2. Magnet apertures \& beam envelopes : (i) The $95 \%$ contour (dashed) with nominal MI beam parameters of $40 \pi$ emittance and $\delta \mathrm{p}_{95} / \mathrm{p}=7 . \mathrm{E}-4$, and ; (ii) the $100 \%$ envelope (solid) for $500 \pi$ emittance $\& \delta \mathrm{p}_{100} / \mathrm{p}=28 . \mathrm{E}-4$, corresponding to the Main Injector aperture at $120 \mathrm{GeV} / \mathrm{c}$.

For the optics solution illustrated in Figure $1, \beta_{x}=\beta_{y}=19.2 \mathrm{~m} \& \alpha_{x}=\alpha_{y}=0$ at the target, which, for a $40 \pi$ beam gives a spot size of $\sigma_{X}=\sigma_{y}=1.00 \mathrm{~mm}$. Since the MI emittance at 120 $\mathrm{GeV} / \mathrm{c}$ is currently measured to be only $\sim 20 \pi$, and it is not well known how this might evolve in the future, it is important that the final focus module be sufficiently robust to produce the desired spot size over a wide range of possible extracted MI emittances. Figure 3 shows the tuning curves for quads Q118 $\rightarrow$ Q121 which fix the spot size as a function of emittance. Across this spectrum of solutions $\beta_{\mathrm{y}}(\max )$ at $\mathrm{Q} 120$ never exceeds $\approx 140 \mathrm{~m}$.

The tuning curves of Figure 3 represent just one, simple, example of the versatile tuning range of the final focus. The advantages of a modular optics design are evident - variation of the MI extracted beam parameters can be accommodated just by tuning the final focus quadrupoles \& does not involve alterations to settings in the remaining bulk of the line. 


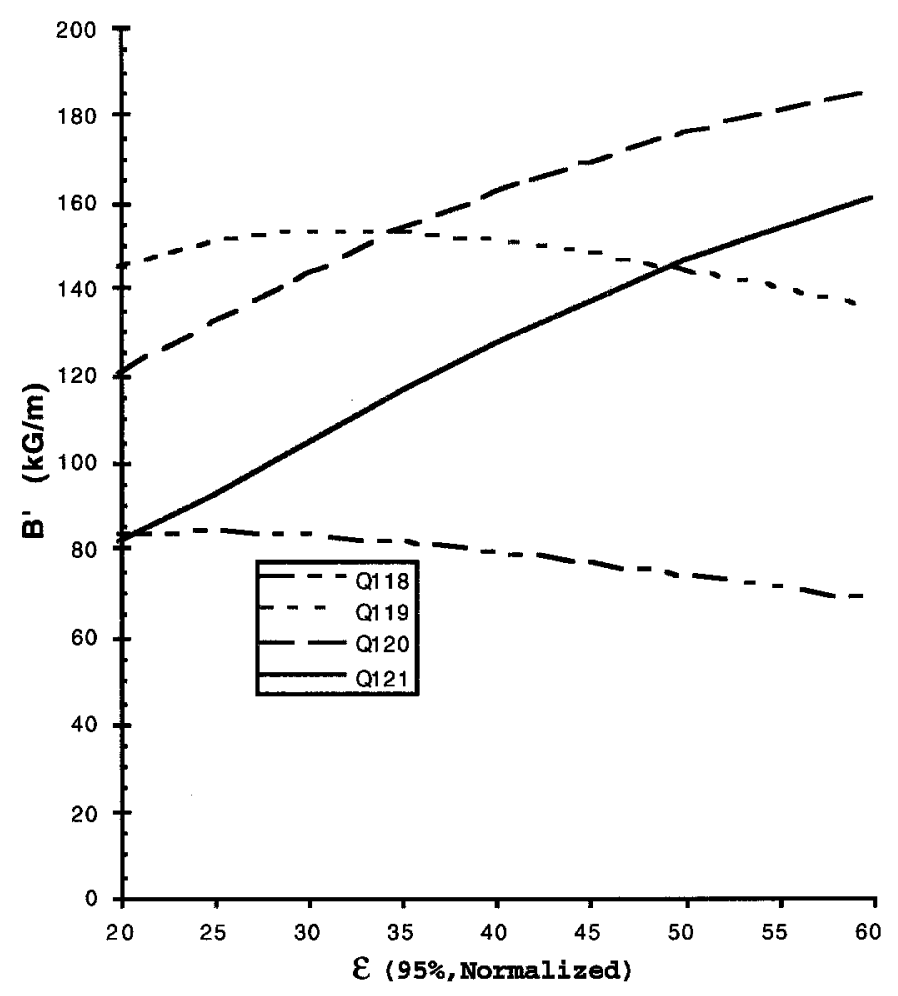

Figure 3. The Q118 $\rightarrow \mathrm{Q} 121$ gradients that produce $\sigma_{\mathrm{X}}=\sigma_{\mathrm{y}}=1.0 \mathrm{~mm}$ at the target as beam emittance varies.

By re-tuning all 6 of the final quadrupoles it is also possible to maintain $\eta_{x}=\eta_{y}=0$ at the target across the $20 \pi \rightarrow 60 \pi$ emittance range considered. Figure 4 illustrates the resulting lattice function for the endpoint emittances. Beam size is clearly well controlled.

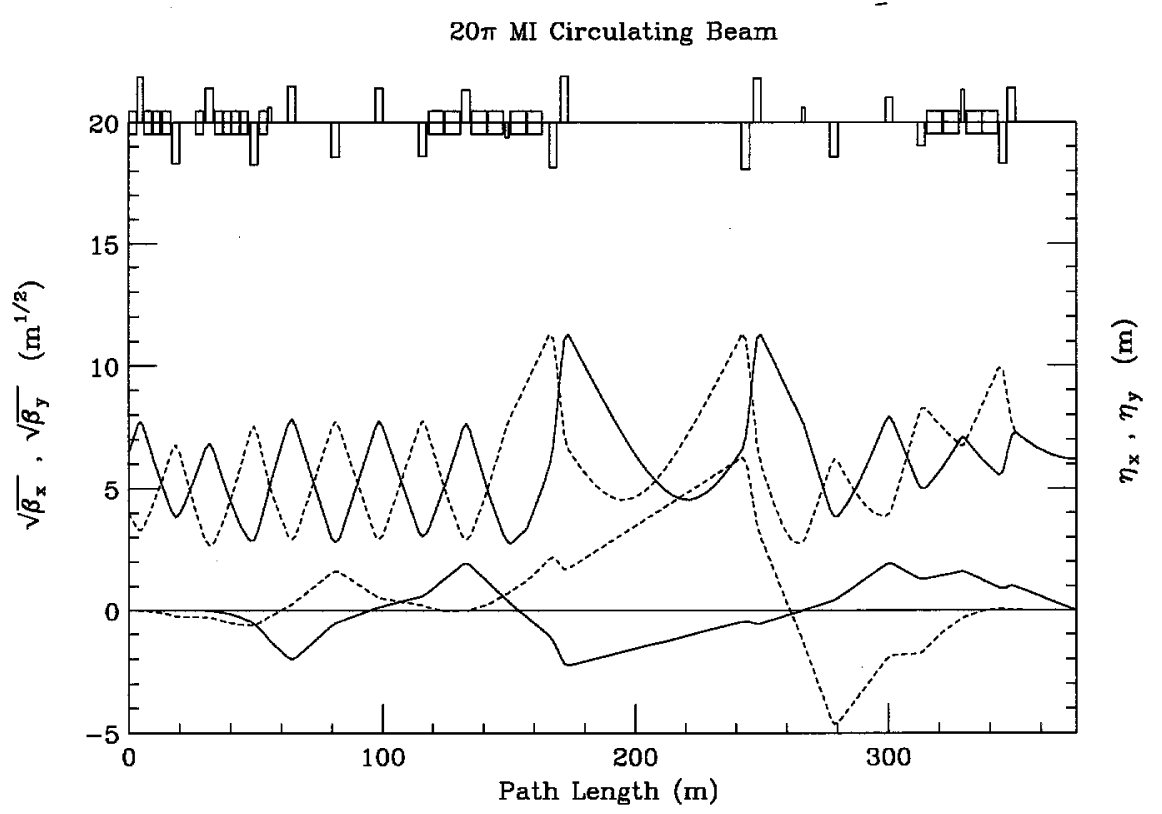




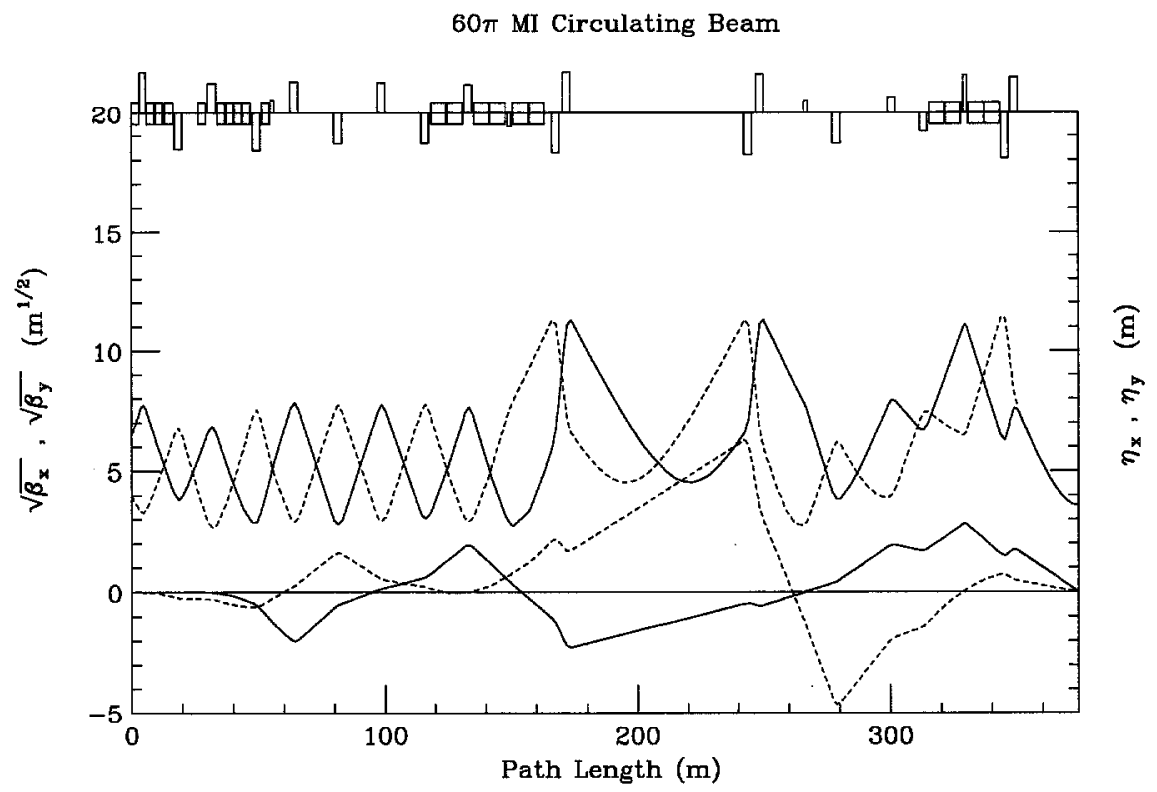

Figure 4. Lattice functions for $20 \pi \& 60 \pi$ beams with the $6 \mathrm{FF}$ quadrupoles tuned to eliminate dispersion at the target while maintaining the $\sigma_{\mathrm{X}}=\sigma_{\mathrm{y}}=1.0 \mathrm{~mm}$ nominal spot size.

The sensitivity of the optics to different error sources has been studied in simulations. Assigning random gradient errors of $\sigma\left(\Delta \mathrm{B}^{\prime} / \mathrm{B}^{\prime}\right)=25 \mathrm{E}-4$ to the 21 quadrupoles in the line, Figure 5 shows the relative $\beta$-waves resulting from 20 random generator seeds. The $\beta$-wave accumulates progressively down the line of course, but $\Delta \beta / \beta$ never exceeds $\approx 10 \%$, which translates into a maximum increase in beam size of $\approx 5 \%$. This feature is confirmed by the results shown in Figure 6 where, for the same 20 seeds, the change in $\sigma$ 's are demonstrated to be less than $\approx 0.10 \mathrm{~mm}$. At the target the maximum changes in beam size are on the order of on'y $0.05 \mathrm{~mm}$.

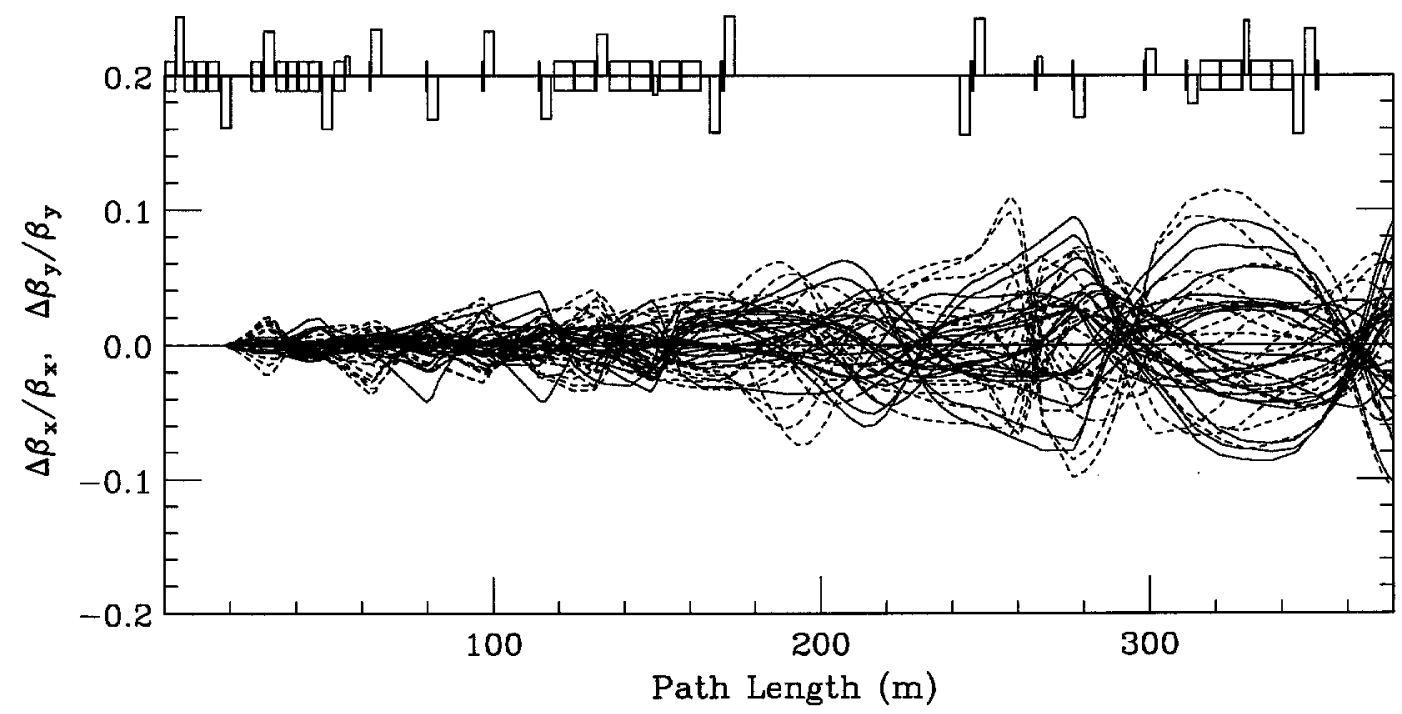

Figure 5. The effect on $\beta$ due to random gradient errors with $\sigma\left(\Delta \mathrm{B}^{\prime} / \mathrm{B}^{\prime}\right)=25 \mathrm{E}-4$. 


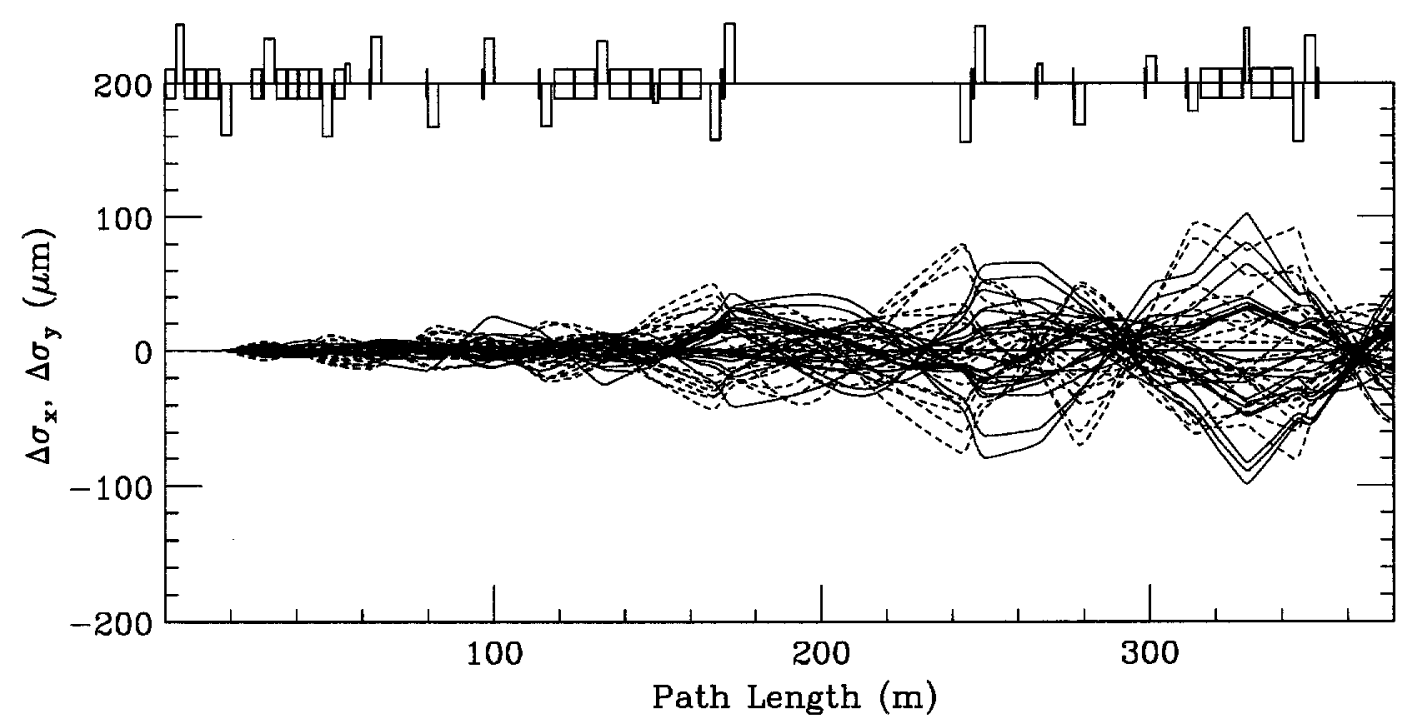

Figure 6. Beam size variation resulting from random gradient errors.

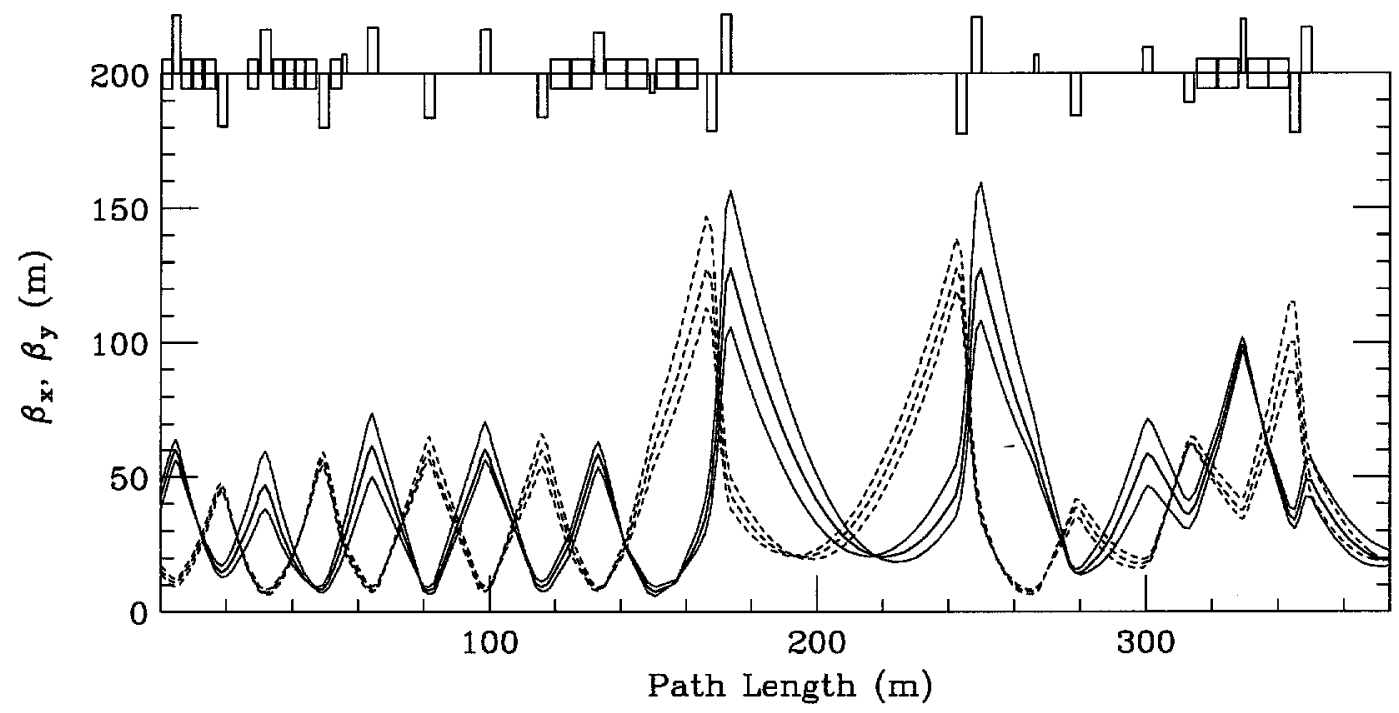

Figure 7. $\beta$-waves due to $\pm 10 \%$ injection optic errors.

Optical errors also arise from discrepancies between the assumed ideal $\&$ true MI lattice functions. Figure 7 shows the $\beta$-envelopes that result from $\pm 10 \%$ variations in the nominal $\beta_{\mathrm{x}} \& \beta_{\mathrm{y}}$ injection values $^{4}$. The MI-end matching section can be re-tuned to eliminate these mismatches, but it can also be seen that this might not be strictly necessary - the maximum $\beta$ 's are sufficiently wellbehaved that no aperture problems arise, and the small residual mismatch at the target can be corrected with the 4 final-focus quadrupoles.

\footnotetext{
4 There is reason to believe that MI $\beta$ errors are corrected (or certainly can be) to $<5 \%$ through tuning of the $53^{\text {rd }}$ -harmonic quadrupole circuits. For details, see; J.A. Johnstone, "A Numerical Simulation of Resonant Extraction", MI-0095, 1993.
} 


\section{Trajectory Correction}

Every focusing element in the line has a BPM associated with it, and both horizontal \& vertical BPM's are assigned to the first quad, Q101. Every quadrupole, with the exceptions of Q101 \& Q104, also has a MI-style dipole corrector nearby. Space limitations at Q101 preclude installing a corrector, while at Q104 a corrector would be redundant with correctors in both planes already nearby at Q103 \& Q105. Orbit correction is an issue which, of course, must be addressed by any beamline, but for the ultra-clean transport requirements of NuMI it is critical that precise position control is available throughout the line.

Correction of central trajectory errors has been simulated with dipole field errors and random misalignments (including BPM's) assigned to the beamline elements. Suitable error values are $\sigma(\Delta \mathrm{x}, \Delta \mathrm{y})=0.25 \mathrm{~mm}, \sigma\left(\psi_{\mathrm{roll}}\right)=0.50 \mathrm{mr}$, and $\sigma(\Delta \mathrm{B} / \mathrm{B})=10 \mathrm{E}-4$. Figure 8 shows the deviations from the central trajectory arising from 20 random error seeds. The uncorrected offsets in the line are $\Delta x(\mathrm{rms})=2.63 \mathrm{~mm}, \Delta \mathrm{x}(\max )=13.03 \mathrm{~mm}$, and $\Delta \mathrm{y}(\mathrm{rms})=2.33 \mathrm{~mm}, \Delta \mathrm{y}(\max )=11.90 \mathrm{~mm}$.

That the deviations are virtually the same in the 2 planes indicates that quadrupole misalignments are the dominant sources of errors. A $15 \mathrm{~T} / \mathrm{m}$ quadrupole displaced transversely by $0.25 \mathrm{~mm}$ produces a kick $\sim 30 \mu \mathrm{r}$, which is larger than the error angle created by $\Delta \mathrm{B} / \mathrm{B}=10$.E- 4 in any of the line's dipoles. In addition, there are more quadrupoles than dipoles.

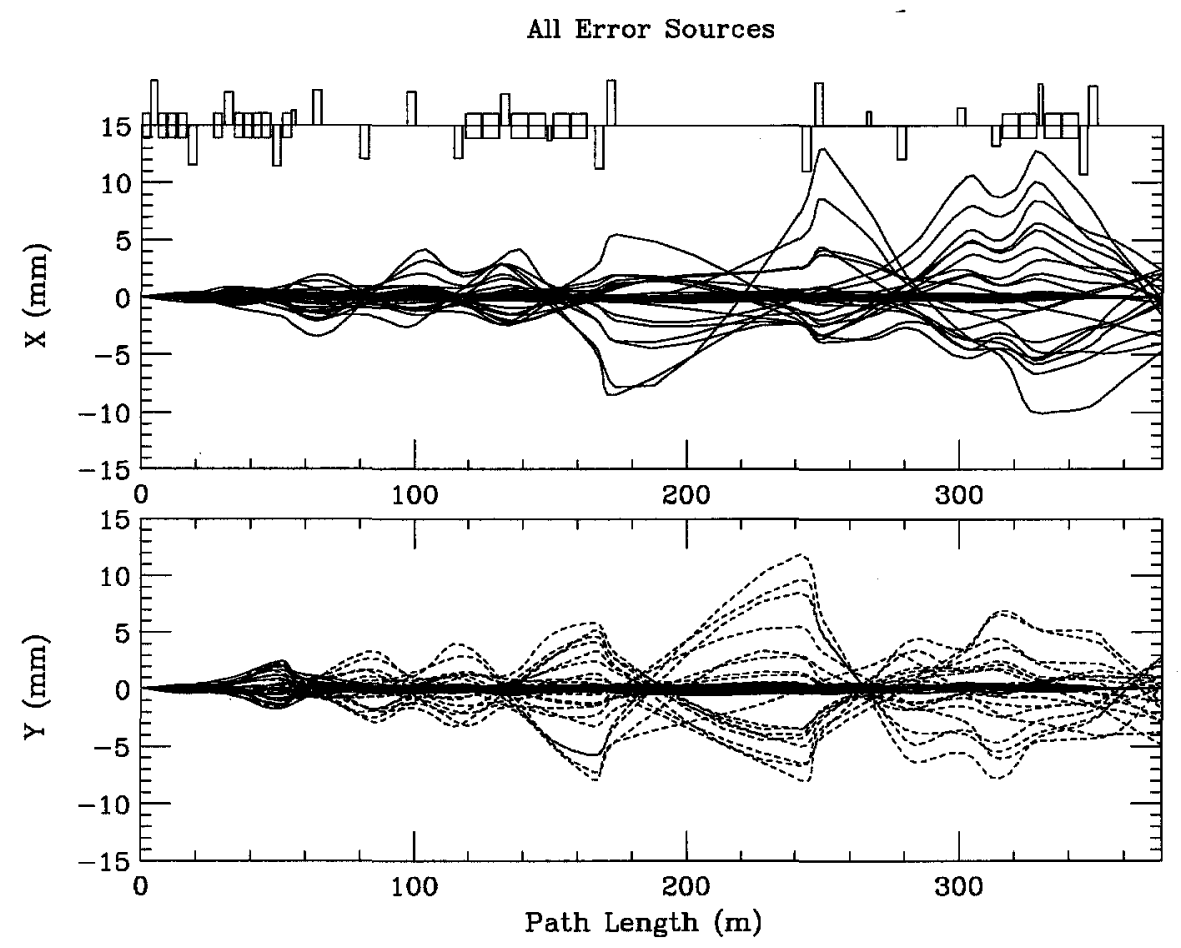

Figure 8. Uncorrected \& corrected trajectories with random misalignments and dipole field errors. 
The corrected orbits are also shown in Figure 8 (in red). After correction the trajectory deviations are reduced to $\Delta x(\mathrm{rms})=0.22 \mathrm{~mm}, \Delta x(\max )=0.85 \mathrm{~mm}, \Delta y(\mathrm{rms})=0.36 \mathrm{~mm}, \Delta \mathrm{y}(\max )=2.44$ $\mathrm{mm}$, and the beam position and angle at the target are tuned to $\Delta x=\Delta y=0 \mathrm{~mm}, \Delta \mathrm{x}^{\prime}=\Delta \mathrm{y}^{\prime}=0 \mu \mathrm{r}$. Maximum x \& y deviations occur at Q102 \& Q103, respectively. This is simply a reflection of the fact that position control at those locations requires tuning the MI kickers, Lambertsons \& Cmagnet — refinements not included in the present simulations.

The strengths required for trajectory correction are $\theta_{X}(\mathrm{rms})=27.57 \mu \mathrm{r}, \theta_{X}(\max )=91.55 \mu \mathrm{r}$, and $\theta_{\mathrm{y}}(\mathrm{rms})=32.00 \mu \mathrm{r}, \theta_{\mathrm{y}}(\max )=95.21 \mu \mathrm{r}$. Horizontal values are comfortably below the $150 \mu \mathrm{r}$ design value available at $120 \mathrm{GeV} / \mathrm{c}$. The maximum vertical kick, however, exceeds the maximum $75 \mu \mathrm{r}$ attainable from a MI-style vertical corrector. Possible solutions are to re-align magnets, install 2 vertical correctors at the bad locations, or install rolled horizontal correctors.

\section{Summary}

The NuMI beamline discussed here is a modular optics design, characterized by 4 sections: MI $\rightarrow$ beamline matching; periodic FODO cells; a special insertion to traverse the carrier pipe, and; a versatile final focus section to produce the desired spot-size on the target. The use of 21 quadrupoles ensures that beam size is constrained within acceptable bounds throughout the line $\beta<60 \mathrm{~m}$ in the MI matching section \& FODO cells, $\beta<125 \mathrm{~m}$ in the doublets of the carrier pipe insertion, and $\beta<100 \mathrm{~m}$ in the final focus. Lattice functions of the NuMI design are not unusually sensitive to errors arising either from MI optical mismatches or gradient errors, and are completely correctable through the 2 matching sections. Aperture studies indicate that the line is able to transport the worst quality beam that the Main Injector might provide. Dipole correctors at 19 of the 21 focusing centers are available to provide high-quality orbit control \& further ensure that the NuMI line meets the stringent requirements for environmental protection.

\section{ACKNOWLEDGMENTS}

The author is pleased to thank Tom Kobalarcik \& Mike Syphers for their useful inputs \& many lively conversations.

\section{$\Omega$}

